\title{
Quarante-cinq ans de JCPH : jamais fermé et en constante rénovation
}

\author{
par Mary H H Ensom
}

$\mathrm{E}$ n tant que grande consommatrice de vêtements ou « mordue de la mode ", j'ai parfois été déçue d'arriver face à une affiche qui indique : «Fermé pour rénovation ". À titre de consommateurs d'articles du JCPH, nos lecteurs ne doivent pas se retrouver dans la même situation. Alors que nous célébrons cette année 45 ans de publication ininterrompue, nous nous préparons à soumettre une demande auprès de la National Library of Medicine (NLM) pour l'indexation dans MEDLINE, l'an prochain. Au fur et à mesure de ce processus, $j$ 'ai pris conscience de l'immense valeur qu'offre le JCPH aux lecteurs du Canada et d'ailleurs dans le monde alors que ceux-ci poursuivent leurs quêtes personnelles et institutionnelles de perfectionnement. De son côté, le JCPH s'est aussi perfectionné au cours des dix dernières années (depuis la dernière demande d'indexation du Journal à la NLM). Voici quelques exemples des améliorations.

\section{PORTÉE ET RAYONNEMENT}

Le JCPH traite principalement de sujets biomédicaux centraux. Alors qu'il n'était que national en 2004 (lorsque seul le format papier était offert), le rayonnement du Journal s'est étendu et ce dernier a maintenant une vocation internationale :

- Plus de 40 organisations et bibliothèques étrangères sont abonnées à la version papier et plus de 60 manuscrits provenant de 22 pays autres que le Canada nous ont été soumis depuis 2007.

- Le comité du Journal compte maintenant trois rédacteurs adjoints provenant de l'étranger : Jim Tisdale des États-Unis, Christine Bond du Royaume-Uni et Rebekah Moles de l'Australie. Ensemble, ils apportent une perspective internationale au Journal. Cynthia Jackevicius, aussi des États-Unis, se joindra à notre équipe en janvier 2015.

En 2010, la demande d'archivage du JCPH dans PubMed Central a été acceptée. Depuis la parution du numéro de janvierfévrier 2013, Multimed Inc., notre partenaire d'hébergement Web, a envoyé des courriels à différents utilisateurs les informant chaque fois qu'un nouveau numéro en ligne était publié : tous les lecteurs du site Web ( $n=11043$ en novembre 2014), et toutes les personnes abonnées et membres de la Société canadienne des pharmaciens d'hôpitaux (SCPH) $(n=5262)$.

\section{QUALITÉ ET TYPES DE CONTENU}

Depuis 2004, les catégories de manuscrits se sont diversifiées afin de correspondre à une classification plus pratique du contenu, particulièrement en ce qui a trait à l'évolution de l'exercice de la pharmacie clinique. Les anciennes options plus larges de catégories ont récemment été redéfinies afin d'inclure : Recherche originale, Articles de synthèse, Innovations dans la pratique de la pharmacie (Pratique clinique, Enseignement de la pharmacie et Pharmacie sociale et administrative), Observations cliniques, Le pour et le contre, Éditoriaux, Commentaires, Lettres de recherche, Correspondance, et critiques de Livres et autres médias. Nous demandons maintenant officiellement des examens systématiques et des éditoriaux en collaboration spéciale par des experts du Canada et de l'étranger.

En 2013, les soumissions se rangeaient dans les catégories suivantes : recherche originale (31\%), articles de synthèse $(6 \%)$, pratique clinique (10\%), enseignement de la pharmacie (1\%), pharmacie sociale et administrative (5\%), observations cliniques (7\%), débat (c.-à-d. Le pour et le contre) (13\%), éditoriaux (6\%), commentaires (6\%), lettres de recherche/correspondance (11\%) et critiques de livre (2\%). Nous nous sommes efforcés d'accroître le contenu scientifique et clinique, et de réduire le contenu non scientifique. La portée linguistique a aussi augmenté, passant de deux articles publiés en français en 2007 à douze en 2013.

En 2007, nous avons lancé la chronique très appréciée « Le pour et le contre " dans laquelle des pharmaciens travaillant dans des établissements de santé débattent de sujets liés à la pratique ou d'ordre thérapeutique qui sont d'intérêt pour la profession. 
En 2011, le conseil de la SCPH a approuvé la politique officielle du JCPH sur l'indépendance éditoriale. La portion non scientifique du Journal ne représente désormais pas plus de $10 \%$ du nombre de pages publiées. Les nouvelles de la Société paraissent maintenant dans l'eBulletin et le Rapport de l'équipe présidentielle a été remplacé par un Commentaire qui est axé sur la place que la SCPH et ses activités prennent dans les enjeux contemporains du monde de la pratique de la pharmacie en établissements de santé.

Afin de favoriser l'intérêt des pharmaciens pour la recherche et de les aider à développer leurs compétences dans ce domaine, nous avons lancé en 2014 une série de deux ans intitulée "Initiation à la recherche ». Les articles, rédigés par des experts sur leurs sujets respectifs et présentés de manière à rendre la matière facilement assimilable, visent à encourager et à soutenir les novices. Le Journal travaille aussi à l'élaboration d'une série sur la pratique pharmaceutique internationale.

Au fil des années, nous n'avons cessé de mettre à jour nos Directives à l'intention des auteurs afin de nous assurer qu'ils respectent les lignes directrices de la NLM quant aux mentions de conflit d'intérêts, au consentement éclairé, aux droits de la personne et à l'animalisme.

\section{QUALITÉ DU TRAVAIL ÉDITORIAL}

Depuis 2004, alors que le $J C P H$ ne disposait que d'un rédacteur en chef et de six rédacteurs adjoints, nous avons agrandi notre comité de rédaction qui compte maintenant une rédactrice en chef et dix rédacteurs adjoints (consultez aussi la section "Portée et rayonnement»). Tous les membres du comité de rédaction sont très respectés et ont acquis une renommée internationale pour leur travail portant sur les aspects scientifiques, cliniques, administratifs ou pédagogiques de la pharmacie. Leurs réalisations en termes de textes publiés ainsi que de leurs activités d'érudition sont impressionnantes et tous possèdent une longue expérience à titre de réviseurs ou de rédacteurs.

Au début de décembre 2014, nous avions 886 réviseurs (dont 49 réviseurs francophones) dans notre base de données et 172 d'entre eux résidaient à l'extérieur du Canada. De plus, leur nombre ne cesse d'augmenter. Enfin, les réviseurs pour chaque manuscrit reçoivent maintenant des copies anonymes des commentaires fournis par les autres réviseurs.

Nos données statistiques montrent une amélioration du temps moyen entre la soumission initiale d'un manuscrit et la première prise de décision (passant de 76 jours en 2007 à 43 jours en 2013), et celui compris entre la soumission initiale et la décision finale (passant de 149 jours en 2007 à 78 jours en 2013). D'ailleurs, en 2013, le temps moyen entre la soumission initiale et la publication était de 3,8 mois.

\section{QUALITÉ DE LA PRODUCTION}

En 2004, le Journal était seulement publié en format papier. En 2007, nous avons lancé eJournalPress (eJPress), un système Web de suivi et de soumission des manuscrits de même que de révision par les pairs. Puis, en avril 2009, nous avons lancé une plateforme de publication en ligne qui utilise l'Open Journal Systems du Public Knowledge Project et dont la conception du site, le téléchargement des articles et le soutien technique sont assurés par Multimed Inc.

De plus, en 2009, nous avons accru le nombre annuel de numéros, passant de cinq à six. Actuellement, les abonnés reçoivent la version papier et la version électronique du Journal, mais à partir de 2015, seule la version en ligne sera offerte.

Multimed Inc. s'est assuré que le JCPH satisfait à toutes les conditions de publication électronique, avec le dépôt des articles dans PubMed Central au moment de la publication, un embargo de six mois sur l'accès libre et un accès immédiat au contenu sur le site Web de l'éditeur sous condition d'une licence admissible.

\section{LECTORAT}

Notre lectorat est maintenant composé de pharmaciens cliniciens, d'enseignants et de précepteurs, d'administrateurs de pharmacie d'hôpital, et de chercheurs du Canada et d'ailleurs dans le monde.

J'ai le plaisir d'affirmer que ce public (les lecteurs et utilisateurs $\mathrm{du} J C P H)$ ne sera pas déçu : après 45 ans, le Journal n'est jamais fermé, mais il est en constante rénovation!

Mary H H Ensom, B. Sc., Pharm. D., FASHP, FCCP, FCSHP, est professeure, Faculté des sciences pharmaceutiques, et boursière universitaire distinguée, The University of British Columbia; et spécialiste en pharmacie clinique, Service de pharmacie, Children's and Women's Health Centre of British Columbia, Vancouver (Colombie-Britannique). Elle est également rédactrice en chef du JCPH.

Intérêts concurrents : Aucun déclaré.

Adresse de correspondance :

Dre Mary H H Ensom

Pharmacy Department OB7

Children's and Women's Health Centre of British Columbia

4500 Oak Street

Vancouver BC V6H 3N1

Courriel : ensom@mail.ubc.ca 